\title{
Biomarcadores en líquido cefalorraquídeo de pacientes con enfermedad de Alzheimer prodrómica
}

\author{
José Antonio Monge Argilés', Carlos Muñoz Ruiz², José Sánchez Payá3, \\ Francisco Javier Montoya Gutiérrez ${ }^{1}$, Enrique Rodríguez Borja ${ }^{2}$ y Carlos Leiva Santana ${ }^{1}$ \\ 'Servicio de Neurología. \\ 2 Laboratorio de Inmunología. \\ 3 Servicio de Medicina Preventiva. \\ Hospital General Universitario de Alicante.
}

\section{Resumen}

Introducción: La enfermedad de Alzheimer (EA) prodrómica es un concepto reciente que incluye la presencia de un deterioro cognitivo leve de tipo amnésico (DCL-a) y la alteración en determinadas pruebas complementarias. Objetivo: Comprobar la existencia de alteraciones en los biomarcadores del líquido cefalorraquídeo (LCR) en aquellos de nuestros pacientes con DCL-a que en el primer año postpunción lumbar desarrollaron la EA. Materiales y métodos: Inicialmente, incluimos 36 pacientes con DCL-a, procedentes de la consulta de deterioro cognitivo de nuestro hospital (criterios de Petersen, 2006) y 24 sujetos control. Por medio de la tecnología xMAP ${ }^{\circledR}$ Luminex se obtuvieron los niveles de las proteínas $A \beta_{1-42}$, T-tau y P-tau $181 \mathrm{p}$ en el LCR, mediante los reactivos INNO-BIA Alz-bio 3 (Innogenetics), y los cocientes T-tau / A $\beta_{1-42}$ y P-tau $181 \mathrm{p} / \mathrm{A} \beta_{1-42}$. Las muestras de 19 pacientes se analizaron por duplicado. Resultados: Los coeficientes de correlación intraclase para cada uno de los tres biomarcadores tenían valores superiores a 0,95. En los doce meses posteriores a la PL, 14 pacientes (38\%) con DCL-a progresaron a EA (criterios NINCDS-ADRDA). Estos últimos presentaban diferencias estadísticamente significativas con respecto a los sujetos control en todos los parámetros estudiados: $A \beta_{1-42}(285,37 \pm 73,36$ frente a $365 \mathrm{pg} / \mathrm{ml}, \mathrm{p}<0,02)$, T-tau ( 88,3 $\pm 40,82$ frente a 53,21 $\pm 23,38 \mathrm{pg} / \mathrm{ml}, \mathrm{p}<0,002)$, P-tau $181 \mathrm{p}(66,44 \pm 23,87$ frente a $31,25 \pm 13,74 \mathrm{pg} / \mathrm{ml}, \mathrm{p}<0,02)$, cociente T-tau / $\mathrm{A} \beta_{1-42}(0,34 \pm 0,19$ frente a $0,15 \pm 0,08$, $\mathrm{p}<0,001)$ y cociente $\mathrm{P}-\mathrm{tau}_{181 \mathrm{p}} / \mathrm{A} \beta_{1-42}(0,25 \pm 0,12$ frente a 0,09 $\pm 0,06, p<0,0001)$. Conclusión: Como viene mostrando la bibliografía médica reciente, nuestra experiencia también pone de manifiesto unas claras alteraciones en los biomarcadores del LCR en los pacientes con EA prodrómica.

(Alzheimer. Real Invest Demenc. 2011;49:19-25)

Palabras clave: biomarcadores, enfermedad de Alzheimer, deterioro cognitivo leve, proteína $A \beta_{1-42}$, proteína T-tau, proteína $\mathrm{P}-\mathrm{tau}_{181 \mathrm{p}}$.

\section{Abstract}

Introduction: The prodromal Alzheimer's disease $(A D)$ is a new concept that include an amnesic mild cognitive impairment (MCIa) and alterations in some complementary tests. Objective: To test the presence of alterations in the cerebrospinal fluid (CSF) biomarkers in our MCI-a patients who developed AD in the first year after the lumbar puncture. Method: 24 control subjects and 36 MCI-a patients were included from the local hospital memory clinic. Using INNO-BIA Alzbio-3 reagents from Innogenetics, we quantified CSF $A \beta_{1-42}$, T-tau and P-tan $181 p$ proteins, and calculated the ratios T-tau / $A \beta_{1-42}$ y P-tau 181p / $\beta_{1-42}$. This project was approved by the local Ethical Committee. Results: One year after the lumbar puncture, 14 MCI patients (38\%) developed AD. These patients showed lower $A \beta_{1-42}$ protein levels $(285.37 \pm 73.36$ vs. $365.21 \pm 112.53 \mathrm{pg} / \mathrm{ml}, p<0.02)$ and higher T-tau protein $(88.3 \pm 40.82$ vs. $53.21 \pm 23.38 \mathrm{pg} / \mathrm{ml}, p<0.002)$, P-tau $181 \mathrm{p}$ protein ( $66.44 \pm 23.87$ vs. $31.25 \pm 13.74 \mathrm{pg} / \mathrm{ml}, p<0.02)$, T-tau $/ A \beta_{1-42}$ ratio $(0.34 \pm 0.19$ vs. $0.15 \pm 0.08, p<0.001)$ and $P$-tan ${ }_{181 p} / A \beta_{1-42}$ ratio $(0.25 \pm 0.12$ vs. $0.09 \pm 0.06, p<0.0001)$ than the control subjects. Conclusion: In our experience, as the recent literature, it exist the very clear alterations of CSF biomarkers in patients with prodromal $A D$.

(Alzheimer. Real Invest Demenc. 2011;49:19-25)

Keywords: CSF biomarkers, Mild cognitive impairment, Alzheimer's disease, A $\beta_{1-42}$ protein, T-tau protein, P-tau 181p protein. 


\section{Introducción}

Los efectos devastadores de la enfermedad de Alzheimer (EA) han llevado a la comunidad científica a la búsqueda de su diagnóstico precoz. Para ello, y desde hace unos años, se ha acuñado el término EA prodrómica ${ }^{1}$, con la finalidad de facilitar la investigación y la aplicación de tratamientos actuales o futuros. Estos criterios diagnósticos de EA prodrómica se han revisado recientemente ${ }^{2}$, pero siguen manteniendo las condiciones de la primera publicación, es decir, la presencia de un deterioro cognitivo leve de tipo amnésico (DCL-a), que se acompaña de una o varias pruebas complementarias alteradas. Entre estas últimas, destaca la cuantificación de las proteínas $A \beta_{1-42}$, T-tau y P-tau 181 p en el líquido cefalorraquídeo (LCR), que viene demostrando desde hace años su capacidad para predecir el desarrollo de EA en los pacientes con DCL ${ }^{3-5}$.

Nuestro objetivo fue comprobar la existencia de alteraciones en los biomarcadores del LCR en aquellos de nuestros pacientes con DCL-a que en el primer año tras la punción lumbar (PL) desarrollaron EA, y que, por tanto, se podían considerar pacientes con EA prodrómica.

\section{Materiales y métodos}

\section{Diseño del estudio}

Estudio de casos y controles.

\section{Sujetos a estudio}

Inicialmente, se incluyeron 36 pacientes con DCL-a diagnosticados según los criterios de Petersen de $2006^{6}$. Todos ellos pertenecían a la consulta de deterioro cognitivo del Hospital General Universitario de Alicante,

Recibido para su publicación: 8 de marzo de 2011. Aceptado para su publicación: 22 de marzo de 2011. Correspondencia: J. A. Monge Argilés.

E-mail:monge_jos@gva.es y a algunos de los cuales se les seguía desde hacía varios años. Su estudio incluye la exploración física y la neurológica, un estudio neuropsicológico, la escala de depresión de Yesavage, la analítica sanguínea, la resonancia magnética nuclear (RNM) cerebral (en 5 casos, tomografía computarizada [TC] cerebral) y la PL. Los casos de estos pacientes se siguen a través de la consulta externa con revisiones cada 6 meses, como mínimo. En éstas, se valora la evolución a EA, según los criterios NINCDS-ADRDA7 (National Institute of Neurological and Communicative Disorders y Alzheimer's Disease and Related Disorders Association), y los pacientes se incluyen en algún estadio de la escala de deterioro global (GDS).

Por otra parte, se incluyeron 24 sujetos control sin quejas subjetivas de memoria ni deterioro cognitivo conocido. Eran pacientes que iban a ser intervenidos mediante raquianestesia de procesos traumatológicos o urológicos no malignos. Una vez firmado el consentimiento informado, se recogieron sus datos clínicos y su analítica sanguínea y, unos días después de la intervención quirúrgica, se les realizó un estudio neuropsicológico. Estos pacientes están invitados a una revisión anual en la consulta de deterioro cognitivo.

\section{Criterios de inclusión}

Pacientes con DCL-a mayores de 55 años. Para la inclusión en el estudio y realización de la PL firmaron el consentimiento informado. En el grupo control, todos los pacientes tenían una valoración en el test Minimental state examination (MMSE) por encima de 27 y una valoración en el cuestionario del informador de deterioro cognitivo en el anciano (IQCODE) por debajo de 78. El deterioro superior a dos puntos en el MMSE o el aumento en más de 7 puntos en el IQCODE se consideraron como conversión a EA en los DCL-a.

\section{Criterios de exclusión}

La presencia de demencia o cualquier otra enfermedad neurológica, psiquiátrica o internística que pudiera provocar deterioro cognitivo, el tratamiento anticoagulante y la falta de consentimiento informado, 
además de una valoración en la escala de depresión de Yesavage superior a 5 puntos.

\section{Procedimientos}

El neurólogo responsable emitía un diagnóstico de DCL-a puro o multidominio, de acuerdo con los criterios de Petersen ${ }^{6}$. Posteriomente, se realizó el estudio neuropsicológico que incluía el test MMSE, IQCODE, Rey auditory verbal learning, California verbal learning, Trail making y la Escala de depresión de Yesavage. Con estos test se realizó la evaluación de la memoria, lenguaje, funciones ejecutivas, atención y capacidades visuoconstructivas. La alteración de una función se definió como un resultado $\mathrm{Z}$ de $-1,5$ o menor, lo cual es al menos 1,5 desviaciones estándar por debajo de la media de los sujetos control en al menos uno de los test usados para estudiar esa función.

Se realizó neuroimagen en todos los pacientes con DCL (RNM en la mayoría de los casos y únicamente TC cerebral en 5 de ellos) para descartar otras lesiones cerebrales responsables del cuadro clínico.

\section{Obtención y análisis del LCR}

Se realizó entre febrero de 2008 y febrero de 2009. Las muestras se obtuvieron entre las 10 y las 14 horas. En los pacientes DCL-a, la PL la realizó el neurólogo responsable, con una aguja $20 \times 3 \frac{1}{2}$. El LCR se recogió en tubos estándar y fue congelado en menos de una hora a $-80^{\circ}$. En el caso de que el LCR fuera ligeramente sanguinolento era centrifugado antes de la congelación. Previamente a la obtención de los tubos para congelar, se recogía uno para la realización de bioquímica y recuento celular. Cuando el LCR era claramente sanguinolento se descartaba.

El LCR de los sujetos control $( \pm 1 \mathrm{ml})$ se obtuvo en el quirófano por el anestesista que llevaba a cabo la raquianestesia.

Tras la PL, se aconsejaba a todos los pacientes evitar maniobras de Valsalva durante al menos 3 días.

\section{Cuantificación de los niveles de proteínas del LCR A $\beta_{1-42}$, T-tau y P-tau 181 p}

Se realizó usando la tecnología xMAP ${ }^{\circledR}$ Luminex con los reactivos INNO-BIA AlzBio3 de Innogenetics (Gante, Bélgica). Los detalles de esta combinación de reactivos para inmunoensayo y plataforma analítica han sido publicados previamente ${ }^{8}$.

Todas las muestras fueron analizadas de forma simultánea, al final del reclutamiento y de forma ciega para los datos clínicos. Las muestras de 19 pacientes fueron analizadas por duplicado.

\section{Variables del estudio}

Los niveles de proteínas del LCR A $\beta_{1-42}$, T-tau, P-tau ${ }_{181 p}$, así como los cocientes T-tau / A $\beta_{1-42}$ y $\mathrm{P}-\operatorname{tau}_{181 \mathrm{p}} / \mathrm{A} \beta_{1-42}$. Estos últimos cocientes están siendo muy utilizados por diversos autores, ya que parecen reflejar la relación entre la vía amiloide y la vía tau de la enfermedad ${ }^{9}$.

\section{Análisis estadístico}

La fiabilidad de la técnica se ha calculado mediante el coeficiente de correlación intraclase.

Para el análisis del tipo de distribución de cada variable se utilizó el test de Kolmogorov-Smirnov. Para la comparación entre 2 grupos, el test de Student para las variables paramétricas y el U-Mann Whitney para las no paramétricas.

Además, se realizó el análisis de las curvas COR (característica operativa del receptor) para determinar los mejores valores de corte con objeto de la medida de las variables. El mejor valor de corte se definió atendiendo sobre todo a la mayor sensibilidad. Después se obtenía la sensibilidad, la especificidad, el valor predictivo positivo (VPP) y el negativo (VPN) para dicho punto de corte y cada variable.

En todos los contrastes de hipótesis se utilizó el nivel de significación estadística de $\mathrm{p}<0,05$. El análisis estadístico se realizó con el paquete SPSS v. 10.0. 


\section{Criterios éticos}

Los laboratorios farmacéuticos que han becado este proyecto no han tenido ningún papel en el diseño, colección o interpretación de datos, ni en la redacción de este trabajo.

Este proyecto fue aprobado por el Comité Ético de Investigación Clínica del Hospital General de Alicante, tras la contratación de un seguro de responsabilidad civil.

\section{Resultados}

\section{Fiabilidad de la técnica}

En los 19 casos analizados por duplicado el coeficiente de correlación intraclase es del 0,94 para la proteína $A \beta_{1-42}$, del 0,96 para la proteína T-tau y del 0,95 para la proteína P-tau.

\section{Comparación del grupo EA prodrómica frente al grupo de control}

Al estudiar la evolución a demencia de nuestros pacientes en los primeros 12 meses, tras la realización de la PL, observamos que 14 de ellos (38\%) habían progresado a demencia de tipo EA según los criterios NINCDSADRDA. Al comparar estos grupos podemos resaltar:

\section{Características clínico-demográficas}

No existen diferencias significativas en la edad de ambos grupos. Los pacientes con EA prodrómica tenían más antecedentes de depresión; sin embargo, el grupo de control presentaba más factores de riesgo vascular (tabla 1).

\section{Comparación de los niveles de proteínas y cocientes}

Existen diferencias estadísticamente significativas entre todas las variables del estudio, y son mayores los valores

\begin{tabular}{|l|c|c|c|}
\hline & Grupo control & Grupo EA prodrómica & Nivel de significación \\
\hline Número de casos & 24 & 14 & \\
\hline Sexo (M/F) & $9 / 13$ & $3 / 11$ & $\begin{array}{c}\text { Sin diferencias } \\
\text { significativas }\end{array}$ \\
\hline Edad (años) & $73,25 \pm 8,34$ & $73,86 \pm 6,95$ & \\
Media \pm DS & 9 & 3 & \\
\hline Antecedentes: & 18 & 7 & \\
DM & 8 & 4 & \\
HTA & 0 & 3 & \\
HPL & No & No & \\
Depresión & 3 & 2 & \\
\hline Hábitos tóxicos & 7 & 5,8 & \\
\hline Antecedentes & - & $25,71 \pm 5.6$ & \\
Demencia de familiares & 28 & Amnésico: 4 & \\
\hline Años de escolarización & - & A Multidominio: 10 & \\
\hline Inicio de los síntomas & & & \\
(meses) media \pm DS & & & \\
\hline MMSE Folstein inicio & & & \\
\hline Tipo DCL & & & \\
\end{tabular}

Tabla 1. Características clínicas y demográficas de los grupos control y DCL-a

A/: amnésico. DM: diabetes mellitus. DS: desviación estándar. EA: enfermedad de Alzheimer. HPL: hiperlipidemia. HTA: hipertensión arterial. M/F: masculino/femenino. MMSE: test Mini mental state examination. 
en el grupo de EA prodrómica para los niveles de proteínas T-tau, P-tau $181 \mathrm{p}$, así como los cocientes T-tau / $A \beta_{1-42}$ y $P-t_{10 u}{ }_{181 p} / A \beta_{1-42}$. Sin embargo, en este grupo, los niveles de proteína $A \beta_{1-42}$ son menores (tabla 2).

\section{Resultados de las curvas COR}

Los valores de sensibilidad y VPN son superiores al $85 \%$ en todas las variables, excepto los niveles de proteína $A \beta_{1-42}$, así como la especificidad para la proteína P-tau 181 p (tabla 3).

\section{Resultados de las curvas COR (según Shaw y cols.)}

Utilizando los puntos de corte propuestos por estos autores, observamos unos máximos niveles de sensibilidad y VPN para la proteína P-tau 181 p y por encima del $90 \%$ para el cociente $\mathrm{P}-\operatorname{tau}_{181 \mathrm{p}} / \mathrm{A} \beta_{1-42}$. Por su par- te, la especificidad es muy elevada para los niveles de proteínas $A \beta_{1-42}$ y T-tau, así como para el cociente T-tau / $\mathrm{A} \beta_{1-42}$ (tabla 4).

\section{Discusión}

Nuestros resultados confirman los datos recientes de la bibliografía médica, que indican la presencia de EA prodrómica en aquellos pacientes DCL-a con alteraciones en los niveles de biomarcadores en el LCR ${ }^{1,2}$. Las diferencias que existen en todas las variables entre los grupos estudiados dan una gran fiabilidad a nuestros resultados, puesto que tanto la alteración de la vía amiloide como la de la vía tau son importantes para el diagnóstico de $\mathrm{EA}^{9,10}$.

De forma similar a Hansson y cols. ${ }^{10}$, nuestros resultados indican que la presencia de alteraciones en estos biomarcadores tiene una alta sensibilidad para el desa-

\begin{tabular}{|c|c|c|c|}
\hline & Grupo control $(n=24)$ & Grupo EA prodrómica $(n=14)$ & Nivel de significación $(p<0,05)$ \\
\hline $\begin{array}{l}\mathrm{A} \beta_{1-42}(\mathrm{pg} / \mathrm{ml}) \\
\text { Media } \pm \text { DS }\end{array}$ & $365,21 \pm 112,53$ & $285,37 \pm 73,36$ & 0,02 \\
\hline $\begin{array}{l}\text { T-tau (pg/ml) } \\
\text { Media } \pm \text { DS }\end{array}$ & $53,21 \pm 23,38$ & $88,30 \pm 40,82$ & 0,002 \\
\hline $\begin{array}{l}\text { P-tau } 181 \mathrm{p}(\mathrm{pg} / \mathrm{ml}) \\
\text { Media } \pm \text { DS }\end{array}$ & $31,25 \pm 13,74$ & $66,44 \pm 23,87$ & 0,02 \\
\hline $\begin{array}{l}\text { T-tau/ A } \beta_{1-42} \\
\text { Media } \pm \text { DS }\end{array}$ & $0,15 \pm 0,08$ & $0,34 \pm 0,19$ & 0,001 \\
\hline $\begin{array}{l}\text { P-tau } 181 p / A \beta_{1-42} \\
\text { Media } \pm \text { DS }\end{array}$ & $0,09 \pm 0,06$ & $0,25 \pm 0,12$ & 0,0001 \\
\hline
\end{tabular}

Tabla 2. Comparación de las concentraciones de los tres biomarcadores y cocientes entre biomarcadores para los grupos control y pacientes con enfermedad de Alzheimer prodrómica.

DS: desviación estándar. EA: enfermedad de Alzheimer.

\begin{tabular}{|l|c|c|c|c|c|}
\hline Parámetros & $\mathbf{A} \boldsymbol{\beta}_{1-42}$ & T-tau & P-tau & T-tau/A $\boldsymbol{\beta}_{1-42}$ & P-tau/A $\boldsymbol{\beta}_{1-42}$ \\
\hline Valor límite & 307 & 55,8 & 39,7 & 0,18 & 0,12 \\
\hline Sensibilidad (\%) & 64 & 86 & 86 & 86 & 86 \\
\hline Especificidad (\%) & 71 & 67 & 87 & 71 & 75 \\
\hline Valor predictivo positivo (\%) & 56 & 60 & 80 & 63 & 67 \\
\hline Valor predictivo negativo (\%) & 77 & 89 & 91 & 89 & 90 \\
\hline
\end{tabular}

Tabla 3. Resultados de las curvas COR para las diferentes variables utilizadas. 


\begin{tabular}{|l|c|c|c|c|c|}
\hline Parámetros & $\mathbf{A} \boldsymbol{\beta}_{1-42}$ & T-tau & P-tau & T-tau/A $\boldsymbol{\beta}_{1-42}$ & P-tau/A $\boldsymbol{\beta}_{1-42}$ \\
\hline Valor límite (Shaw) & 193 & 93 & 23 & 0,39 & 0,10 \\
\hline Sensibilidad (\%) & 21 & 36 & 100 & 36 & 93 \\
\hline Especificidad (\%) & 87 & 92 & 25 & 96 & 67 \\
\hline Valor predictivo positivo (\%) & 50 & 71 & 44 & 83 & 62 \\
\hline Valor predictivo negativo (\%) & 66 & 71 & 100 & 72 & 94 \\
\hline
\end{tabular}

Tabla 4. Resultados de las curvas COR para las diferentes variables utilizadas, tomando como referencia los valores de Shaw y cols. ${ }^{9}$.

rrollo de EA, así como su normalidad posee una alta fiabilidad para descartar dicho diagnóstico. Estos datos pueden ser muy útiles en la práctica clínica diaria para descartar el desarrollo de EA en pacientes con depresión o que sean difícilmente valorables, desde el punto de vista neuropsicológico.

Actualmente, se aceptan diferentes exámenes complementarios para el diagnóstico de EA prodrómica, a partir de DCL-a ${ }^{1,2}$. Como era de esperar, la combinación de dichas técnicas complementarias aumenta la sensibilidad para el diagnóstico precoz de EA ${ }^{11}$. Sin embargo, las otras dos técnicas propuestas (tomografía por emisión de positrones [PET] y volumetría en RNM) resultan actualmente menos efectivas según su coste que el análisis de biomarcadores en el LCR ${ }^{12}$. Este hecho, en las circunstancias sanitarias actuales de nuestro país, nos parece un elemento decisivo para su uso en el diagnóstico precoz de EA.

Por otra parte, en nuestro estudio podemos observar la alta fiabilidad y reproducibilidad de los resultados con la técnica utilizada. $\mathrm{Al}$ analizar nuestros datos con los puntos de corte publicados por Shaw y cols. ${ }^{9}$, a partir de LCR obtenidos por autopsia de pacientes con EA demostrada, vemos que P-tau muestra una sensibilidad y VPN máximos.

Aunque los resultados obtenidos en nuestras curvas COR no son tan favorables como los suyos, consideramos los valores de dichos autores como referencia, puesto que han sido obtenidos de pacientes con EA definitiva? ${ }^{7}$.

Las mayores limitaciones de nuestro estudio son la muestra reducida y el corto período de seguimiento. Sin embargo, continuamos la observación semestral en nuestro corte de pacientes y anual en el grupo control.

\section{Agradecimientos}

A los Servicios de Anestesia, Urología y Traumatología del Hospital General Universitario de Alicante. A María de los Ángeles Miguelsanz del laboratorio de Inmunología, por la magnífica gestión de las muestras. A Margarita Ruiz Vegara, por su colaboración desinteresada (in memoriam).

A los laboratorios Novartis España y Grunenthal España por el soporte financiero.

\section{Bibliografía}

1. Dubois B, Feldman HH, Jacova C, DeKosky ST, Barberger-Gateau P, Cummings J, et al. Research criteria for diagnosis of Alzheimer's disease: revising the NINCDSADRDA criteria. Lancet Neurol. 2007;6:734-46.

2. Dubois B, Feldman HH, Jacova C, Cummings J, DeKosky ST, Barberger-Gateau P, et al. Revising the definition of Alzheimer's disease: a new lexicon. Lancet Neurol. 2010; 9:1118-27.

3. Diniz BS, Pinto Junior JA, Forlenza OV. Do CSF total tau, phosphorylated tau and beta-amyloid 42 help to predict the progression of mild cognitive impairment to Alzheimer's disease? A systematic review and metaanalysis of the literature. World J Biol Psychiatry. 2008; 9:72-82.

4. Monge Argilés JA, Sánchez Payá J, Muñoz Ruiz C, Pampliega Pérez A, Montoya Gutiérrez J, Leiva Santana C. Biomarcadores en LCR de pacientes con deterioro cognitivo leve: metaanálisis de su capacidad predictiva para el diagnóstico de la enfermedad de Alzheimer. Rev Neurol. 2010;50:193-200.

5. Mitchell A, Monge Argilés JA, Sánchez Payá J. Do CSF biomarkers help clinicians predict the progression of mild cognitive impairment to dementia? Pract Neurol. 2010; 10:202-7.

6. Artero S, Petersen RC, Touchon J, Ritchie K. Revised criteria for mild cognitive impairment: validation within a 
Monge Argilés JA et al. Biomarcadores en líquido cefalorraquídeo de pacientes

con enfermedad de Alzheimer prodrómica

longitudinal population study. Dement Geriatr Cogn Disord. 2006;22:465-70.

7. McKhann G, Drachman DA, Folstein M, Katzman R, Price DL, Stadlan EM. Clinical diagnosis of Alzheimer's disease- report of the NINCDS-ADRDA work group under the auspices of Department of Health and Human Services Task Force on Alzheimer's disease. Neurology. 1984;34:939-44.

8. Olsson A, Vanderstichele H, Andreasen N. Simultaneous measurement of $\beta$-amyloid 1-42 $_{2}$, total tau and phosphorylated tau in CSF by xMAP technology. Clin Chem. 2005; $51: 336-45$.

9. Shaw LM, Vanderstichele H, Knapik-Czajka M, Clark CM, Aisen PS, Petersen RC, et al. Cerebrospinal fluid biomarkers signature in Alzheimer's disease neuroimaging initiative subjects. Ann Neurol. 2009;65:403-13.

10. Hansson $\mathrm{O}$, Zetterberg $\mathrm{H}$, Buchhave $\mathrm{P}$, Londos $\mathrm{E}$, Blennow K, Minthon L. Association between CSF biomarkers and incipient Alzheimer's disease in patients with mild cognitive impairment: a follow-up study. Lancet Neurol. 2006;5:228-34.

11. Vemuri P, Wiste HJ, Weigand SD, Shaw LM, Trojanowski JQ, Weiner MW, et al. MRI and CSF biomarkers in normal, MCI and AD subjects. Predicting future clinical change. Neurology. 2009;73:294-301.

12. Hort J, Bartos A, Pirttila T, Scheltens P. Use of CSF biomarkers in diagnosis of dementia across Europe. Eur J Neurol. 2010;17:90-6. 